\title{
Guías de publicación para los diferentes tipos de artículos médicos
}

\section{Publication guidelines for different types of medical articles}

\author{
Denzil Garteiz Martínez*
}

* Editor en Jefe de la Revista Mexicana de Cirugía Endoscópica. ORCID: 0000-0002-8479-4076.

Los cirujanos, como todos los médicos, tenemos una formación con bases científicas. Sin embargo, comparado con nuestros colegas clínicos, por lo general, nos cuesta mucho trabajo involucrarnos en proyectos de investigación serios y publicar nuestros resultados en revistas académicas. Algunos dirán que es porque somos más prácticos y dependemos más de nuestra experiencia personal, pero quizá también es porque tenemos menos paciencia para escribir y menor tolerancia a ser evaluados por nuestros pares. Vencer estos obstáculos y adquirir estas últimas dos cualidades es indispensable para un autor que pretende que su trabajo sea publicado en una revista médica.

El proceso de publicación de un artículo científico podría compararse, toda proporción guardada, con

Tabla 1: Guías para publicación de diferentes tipos de trabajo científico.

Tipo de artículo

Caso clínico

Estudio observacional

Protocolos de estudio

Ensayo clínico controlado

Revisión sistemática

Estudios de diagnóstico/pronóstico

Guías de práctica clínica

Investigación cualitativa

Estudios preclínicos en modelo animal

Estudios de calidad

Estudios de evaluación económica
Guía

CARE

STROBE

SPIRIT

CONSORT

PRISMA

STARD

AGREE

SRQR

ARRIVE

SQUIRE

CHEERS
Liga

https://www.care-statement.org

https://www.strobe-statement.org

https://www.spirit-statement.org

http://www.consort-statement.org

http://prisma-statement.org

https://www.equator-network.org/reporting-guidelines/stard/

https://www.equator-network.org/reporting-guidelines/ the-agree-reporting-checklist-a-tool-to-improve-reporting-ofclinical-practice-guidelines/

https://www.equator-network.org/reporting-guidelines/srqr/ https://www.equator-network.org/reporting-guidelines/ improving-bioscience-research-reporting-the-arrive-guidelinesfor-reporting-animal-research/

https://www.equator-network.org/reporting-guidelines/squire/ https://www.equator-network.org/reporting-guidelines/cheers/

Tomado de: Enhancing the QUAlity and Transparency of Health Research. Disponible en: https://www.equator-network.org/ 
el desarrollo de una tesis de maestría o doctorado, en donde el editor y los revisores de la revista toman el temido papel del asesor de tesis. Así como el tesista teme y se frustra con cada rechazo o corrección de su asesor, el autor de un artículo suele decepcionarse con los comentarios y sugerencias del equipo editorial de la revista. Sin embargo, existe una diferencia fundamental entre estos dos procesos, y la función del asesor de tesis y del editor de una revista no son iguales. Mientras que una tesis implica documentación extensa y texto mucho más amplio, escribir un artículo para una revista científica requiere de gran capacidad de síntesis y enfoque en el tema principal. Así mismo, mientras que un asesor de tesis es un experto en el tema y sus aportaciones se centran en explotar cada detalle del mismo, un editor debe desmenuzar la información y seleccionar exclusivamente aquella que sea indispensable para cumplir con los criterios de publicación.

Los cirujanos podemos contribuir a la literatura científica de varias maneras y las revistas médicas generalmente nos ofrecen diferentes formatos para publicar. La importancia del tema a presentar y el tipo de investigación realizada determinarán el impacto de la publicación, que puede abarcar desde la descripción de un caso interesante hasta la presentación de un ensayo clínico controlado o una revisión sistemática. Todas son formas válidas para el desarrollo académico y todas ofrecen oportunidades para que los cirujanos cumplamos con nuestra responsabilidad de participar en la educación continua.
Antes de empezar a escribir, el autor debe familiarizarse con las guías prácticas para cada uno de los formatos existentes. No es lo mismo escribir un reporte de caso, que una revisión de la literatura o que un metaanálisis. Estas guías internacionales pueden encontrarse fácilmente en línea (Tabla 1) y, por lo general, cada revista tiene una sección de «Instrucciones para los autores», que sintetiza los puntos clave para cada tipo de artículo. Así mismo, antes de enviar un manuscrito para publicación, el autor debe verificar cada punto de las listas de cotejo proporcionadas en las guías. Esto no sólo facilitará la redacción y dará una estructura adecuada al trabajo, sino que también aumentará las posibilidades de que sea aceptado por la revista. Recordemos que, a diferencia del asesor de tesis, que es experto en el tema, el editor generalmente no hará modificaciones al contenido de la información, pero sí las hará con respecto al formato de presentación y al cumplimiento de los requisitos básicos de publicación. La función del editor no es cuestionar los conocimientos del autor, sino garantizar que su revista publique artículos de calidad, escritos de acuerdo con las guías establecidas.

Con el fin de simplificar el proceso de redacción y revisión de artículos, el comité editorial de la Revista Mexicana de Cirugía Endoscópica pondrá a su disposición las listas de cotejo para cada tipo de artículo, traducidas al español, en la página de la Asociación. Los invitamos a compartir con nosotros sus experiencias y a fomentar entre sus residentes y alumnos la publicación de material científico de alta calidad. 